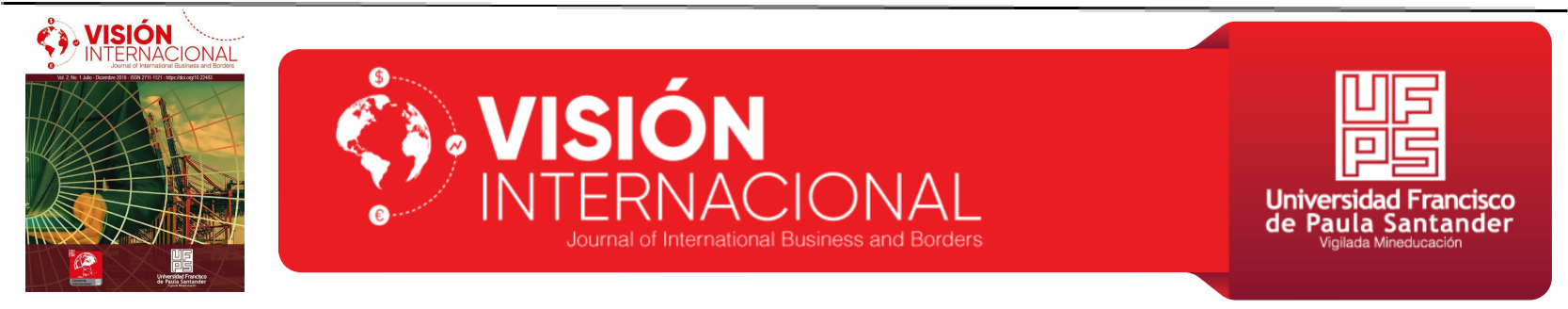

\title{
La importancia de la religion frente a los negocios internacionales
}

The importance of religion against international business

Oscar Arnulfo Mera Ramírez ${ }^{a^{*}}$

$a^{*}$ Estudiante de doctorado en proyecto, ,oscaralnulfomr@ufps.edu.co,orcid.org/0000-0001-9004-55701, Universidad Francisco de Paula Santander, Cúcuta, Colombia

Cómo citar: Mera-Ramírez, O.A (2019) La importancia de la religión frente a los negocios internacionales. Revista Visión Internacional, 2(4), 29-31

Recibido: Enero 7, 2019; Aprobado: Marzo 25, 2019

\begin{tabular}{|c|c|}
\hline & RESUMEN \\
\hline $\begin{array}{l}\text { Comercio Internacional, } \\
\text { Cultura, conservatismo, } \\
\text { religiosidad }\end{array}$ & $\begin{array}{l}\text { El comercio Internacional y en general la forma de negociar, se ha vuelto una } \\
\text { actividad más común y al mismo tiempo cambiante entre los países en el mundo, } \\
\text { hoy en día podemos encontrar mayores niveles de competitividad global } \\
\text { acompañado del predominio de un libre mercado el cual se nutre del intercambio } \\
\text { comercial y de poder satisfacer necesidades propias de cada nación, en algunas } \\
\text { ocasiones puede ser más flexible dicho comercio y en otras puede haber múltiples } \\
\text { complejidades y diversas diferencias que existen en este tema y que son causadas } \\
\text { entre otros aspectos por las políticas que cada gobierno impone según sus } \\
\text { necesidades o intereses, Sistemas económicos diferentes y costumbres Religiosas } \\
\text { que hacen que la política comercial funcione de forma diferente. Es por esto que en } \\
\text { este artículo se presta gran atención a la importancia que tiene dichas culturas o } \\
\text { costumbres en algunos países que son multireligiosos y como esto puede influir en } \\
\text { temas del Comercio y de las relaciones Internacionales. }\end{array}$ \\
\hline
\end{tabular}

\section{ABSTRACT}

Keywords:

International trade and in general the way of negotiating, has become a more common and at the same time changing activity among countries in the world, today

International Trade, Culture, conservatism, religiosity we can find higher levels of global competitiveness accompanied by the predominance of a free market which is It nurtures commercial trade and being able to satisfy the needs of each nation, sometimes this trade may be more flexible and in others there may be multiple complexities and diverse differences that exist on this subject and that are caused, among other aspects, by the policies that each According to their needs or interests, the government imposes different economic systems and religious customs that make trade policy work differently. That is why this article pays great attention to the importance of these cultures or customs in some countries that are multi-religious and how this can influence issues of Commerce and International relations.

\section{Introducción}

*Autor de Correspondencia.

E-mail: oscararnulfomr@ufps.edu.co (c) $(i)$

(Oscar Arnulfo Mera Ramírez)

Peer review is the responsibility of the Universidad Francisco de Paula Santander

This is an article under the license CC BY-ND 
El comercio internacional, es un fiel representante de lo que se puede denominar la globalización en pleno siglo XXI, contiene las mejores vertientes de conectividad, toma de decisiones y por supuesto adaptación a los cambios repentinos e inesperados, tanto de los mercados, como de los consumidores.

¿Pero toda esta facilidad de relaciones interpersonales e interempresariales se ve en algún momento truncada por variables o por así decirlo obstáculos en su haber? Claramente la respuesta es, Sí, ya que en todo el mundo se encuentran una, dos o más religiones en determinadas zonas geográficas, por lo cual esto significa tener diferencias de ámbitos culturales, comerciales, políticos y de consumo que se presentaran al realizar negocios.

Un claro ejemplo que se puede llegar a tener es el de Egipto, una nación de milenaria cultura, de estratégica ubicación al Norte de África y al oeste de medio oriente, con inmensos recursos como reservas de petróleo, la principal producción de algodón del mundo, el canal de Suez y ser uno de los sitios predilectos para el turismo. (Republica Arabe de Egipto, 2012) Su composición religiosa es de la siguiente forma: el $80 \%$ de la población es de fe Islámica conformada por sunníes y chiíes, y el restante $20 \%$ de fe cristiana compuesta por ortodoxos, coptos y maronitas entre otros. (Mateos, 2016)

Observando las consideraciones anteriores, se puede determinar el hecho de que Egipto es un país multireligioso, predominado por el islam, pero con influencias de diversos cultos, tanto así que esto demarca su estilo de consumo de ciertos productos, como lo son los productos derivados del cerdo, el alcohol o incluso el literario. (Minue, 2014) Asimismo, todo negocio que se desee realizar en Egipto debe de ser centrado en el entendimiento de su cultura, como de su religión, tanto desde su aspecto externo como interno, y que ventajas y desventajas proporciona, teniendo todo esto en cuenta, se puede dar un muy provechoso acuerdo comercial con empresas egipcias o potenciales consumidores de los productos a comercializar.

Cabe agregar que podemos tener a consideración en el tema tratado, al joven estado de Israel, vecino del ya mencionado Egipto, que con similitud poseen una muy basta cultura y una economía cada vez más participativa en el ámbito internacional. La economía del estado judío es de una abertura sin igual en medio oriente, pero esto no significa que no existan limitaciones o restricciones con respecto a ciertos productos o contenidos que se lleguen a considerar inapropiados para el consumidor israelí. (Santander Trade, 2018)

Por ello al momento de realizar negocios en Israel no es de extrañarnos que se encuentren solamente judíos, también existen cristianos israelíes y musulmanes de nacionalidad israelí, con requerimientos de consumo muy diferentes a los demás países de medio Oriente.

La relación existente entre la religión y el comercio en Israel es de tal magnitud, que ha generado un nuevo tipo de mercado, el mercado kosher, que básicamente se compone por alimentos orgánicos y de clasificación apta para el consumo del pueblo judío, pero este mercado no solo se limita con consumidores judíos, gran parte de Europa tiene en muy buena consideración, los productos con la certificación kosher, ya que son de excelente calidad y de un cuidado esencial para la salud del ser humano, todo esto derivado de las creencias hebreas nacientes en la Tora. (Freeman, 2011) Otro aspecto sumamente importante de la economía israelí es su nivel de exportación tecnológica, que es apetecida en gran medida por los mercados mundiales, en los ámbitos civiles, como militares, pero con la salvedad de que el comprador debe de tener muy buenas relaciones diplomáticas con el estado judío, todo esto debido a su política de protección frente a futuras amenazas y no verse envueltos en conflictos de intereses. (Datos Macro, 2017)

Asimismo, el desarrollo e innovación que solo se puede encontrar allí, es de un nivel que solo es encontrado en grandes potencias como Estados Unidos o China, ya que la educación es el eje principal para el desarrollo de una 
persona en Israel, así que esto les da gran valor en el mercado tecnológico, pero siempre en miras a la preservación de la cultura $\mathrm{y}$ creencias de origen judío. (Jorda, 2017)

Desde luego el conservatismo y en ciertas ocasiones la influencia de otras culturas, generan ciertos temores en algunos sectores de la sociedad, que pueden fácilmente derivar en discriminación o racismo hacia nuevos agentes de participación cultural.

Muestra de lo anterior esta el caso de Austria, un pequeño estado europeo en mitad de Europa central, con una población de no más 8 millones de habitantes y una extensión geográfica de tan solo $83.773 \mathrm{~km} 2$, pero con un nivel de calidad de vida envidiable para muchos estados de la región. (Gifex, 2006) Con una economía estable, caracterizada por su sostenimiento a base de la creación de pymes, enfocadas en la prestación de servicios y manufacturas, sin dejar de lado la inversión, o sectores tan tradicionales como el industrial, que compone el $25 \%$ del PIB, o el creciente sector agrícola ecológico, que haya el $43 \%$ de sus cultivos dedicados a este tipo de producción sostenible. (Santander Trade, 2018) Todos estos factores le han convertido en uno de los destinos predilectos para los inmigrantes provenientes de África y medio oriente, culturas totalmente opuestas a la austriaca, a pesar de ser un país de apertura comercial y con una composición religiosa del $61 \%$ concentrado en el cristianismo católico, un 6\% de cristianismo ortodoxo, $6 \%$ creyente en el islam y $12 \%$ de ateos. (Ripley Believes, 2012)A pesar de tener esta diversidad religiosa, y de tener el título de primer país en aceptar el islam como religión reconocida en Europa y poseer

Un secularismo bien demarcado, últimamente se ha visto envuelto en escándalos de racismo, con expresiones provenientes de sectores del gobierno, que están en contra de los inmigrantes, y su cultura.

Como claro ejemplo la reciente ley austriaca, que prohibía usar el burka (velo islámico para las mujeres) en los espacios públicos, ya que este representa una influencia directa en la sociedad austriaca. (Zumata, 2017) O su reciente salida del pacto mundial para la inmigración, porque este representaba una amenaza a la soberanía del país alpino, y el documento se encontraba con grandes vacíos como la definición de migración legal o ilegal, además de las limitaciones de las fuerzas de seguridad en las fronteras. (El Mundo, 2018) Estas decisiones se han caracterizado determinantes en la agenda política de Austria, por lo que, al momento de realizar negocios, estos son temas un tanto álgidos y se sobre estima el interés en tratarlos, por su volatilidad y por supuesto un tanto polémicos.

Finalmente se concluye, en la importancia y el entendimiento de las diferencias culturales entre las naciones, y sus implicaciones al momento de hacer negocios, y como no ser imprudente al momento de realizarlos. Existe una gran relevancia entre la relación que existe entre los temas de valores, costumbres, y religiones con el comercio Internacional pues a la hora de hacer alguna negociación no solo hay que tener en cuenta estos aspectos, sino que también se debe incluirse temas como su forma de pensar o de proyectar los negocios, puede ser que signifique lo mismo, pero se les dé una interpretación diferente según sea su religió o cultura

\section{Referencias}

Salas, M.P, (2018). Te explicamos la historia palestina-

israelí. Revista El Definido.

Jorda, C. (2020) Ocho claves del éxito de Israel

en innovación

Revista Sputnink mundo (2020)

U.S. Central Intelligence. (2006). población de Austria

Política económica (2019)

Nuevas estadísticas de la Iglesia en Austria: 
La importancia de la religion frente a los negocios internacionales

cifras estables, con una ligera subida de bautismos (2011) 